\title{
Quel savoir pour quelle citoyenneté ?
}

Débat. Avec Christine Lazarges, René Revol, Marc Coutty, Pierre Boutan, Anne Yvonne Le Dain, Pierre Antonini, Jean-Pierre Obin

\section{Marc Coutty}

\section{(2) OpenEdition}

1 Journals

\section{Édition électronique}

URL : http://journals.openedition.org/trema/1763

DOI : $10.4000 /$ trema. 1763

ISSN : 2107-0997

\section{Éditeur}

Faculté d'Éducation de l'université de Montpellier

\section{Édition imprimée}

Date de publication : 1 octobre 1999

Pagination : 171-189

ISSN : 1167-315X

\section{Référence électronique}

Marc Coutty, «Quel savoir pour quelle citoyenneté ? », Tréma [En ligne], 15-16 | 1999, mis en ligne le 09 novembre 2010, consulté le 15 septembre 2020. URL : http://journals.openedition.org/trema/1763

Ce document a été généré automatiquement le 15 septembre 2020.

Trema 


\section{Quel savoir pour quelle citoyenneté?}

Débat. Avec Christine Lazarges, René Revol, Marc Coutty, Pierre Boutan, Anne Yvonne Le Dain, Pierre Antonini, Jean-Pierre Obin

\section{Marc Coutty}

\section{Débat}

\section{$1 \quad$ Marc COUTTY}

Nous allons clore ces deux journées de débat que vous avez eues autour du thème savoir, langages et citoyenneté... Je laisserai nos invités se présenter les uns après les autres, vous en connaissez sans doute certains déjà... et nous allons essayer pour véritablement clore cette journée en tenant compte de choses qui m'ont été dites préalablement de bien tenir les trois bouts de la réflexion, c'est-à-dire de mettre l'accent, je dirais, sur les conjonctions de coordination afin de ne pas parler pour les uns du savoir, pour les autres du langage, pour les autres de la citoyenneté mais bien d'entrer au cœur de notre problématique et de voir en quoi il y a interaction d'un des termes sur les autres. Monsieur René Revol, du Cercle Condorcet, va, je crois, essayer de nous cadrer un peu le débat, de nous dire en tout cas ce qu'il en pense. Ce que je vous propose, c'est que nous fassions un tour de table avec nos invités. Nos invités diront ce qu'ils pensent du sujet, après quoi il y aura des questions de votre part, si vous souhaitez en poser, et nos invités y répondront. Naturellement tout le monde ne pourra pas répondre à toutes les questions mais nous essayerons de faire au mieux. Je vous passe la parole.

\section{René REVOL}

Eh bien, je vous remercie. Je peux commencer ce débat tout simplement par la réflexion que nous avons eue au Cercle Condorcet de Montpellier sur la citoyenneté. Dans cette réflexion nous avons rencontré la place qu'occupent le savoir et le langage. Je signale quand même que cette réflexion on l'a eue l'année dernière... juste après que l'ombre portée d'un parti xénophobe se soit étendue sur l'exécutif régional. On peut 
difficilement, même dans un colloque universitaire, aborder les problèmes de la citoyenneté sans que les enjeux qui traversent la société " nous interrogent un peu quelque part ». Alors, cette réflexion, nous avons été amenés à la pousser et à voir que, finalement, beaucoup d'éléments que nous avons apportés, découverts, ont été résumés dans la communication précédente qu'à faite Monsieur l'Inspecteur Général Obin tout à l'heure. La définition de la citoyenneté est souvent réduite à la simple civilité, et en fait, c'est une sorte de mutilation profonde de la citoyenneté et c'est peut-être le moyen de ne pas améliorer la civilité que de réduire la citoyenneté à la seule civilité. Et lorsqu'on est amené à réfléchir aux dimensions de la citoyenneté, on en reconnaît trois :

1. il y a premièrement une citoyenneté civique. Ce sont les droits. A ce sujet, très souvent, les droits sont présentés comme «le droit de », et mal présentés, tout se passe comme s'il s'agissait de demander des droits. Ces droits, on ne les demande pas. D'abord on les conquiert puis on les exerce et ce ne sont pas des droits comme il y a un droit commercial, comme il y un code de la route; ce ne sont pas des normes. C'est finalement une responsabilité qu'on exerce, au sein de la société, et on nous donne les moyens de l'exercer. Donc, au fond, quand on réfléchit aux droits, aux droits de l'Homme, on va en parler toute la semaine je crois, il faudrait passer du «droit de » au «droit à » parce qu'il ne s'agit pas simplement d'avoir « le droit d'expression », il s'agit d'avoir « le droit à l'expression ». Donc il n'y a pas, comme l'a déjà très bien expliqué Condorcet - la citation a déjà été faite avant - de droits s'il n'y a pas de capacité à exercer ces droits. Et à ce sujet, cette capacité à exercer « ses » droits, elle ne se fait pas simplement dans le sens où il s'agit de transmettre des moyens. C'est un premier élément, les moyens : les moyens en termes de savoir, les moyens en termes de langage. Il s'agit aussi de faire que ces moyens soient conquis, disons soient acquis, par les citoyens dès leur plus jeune âge; la question se pose finalement sous des formes très différentes dès l'enfance.

Naturellement, la citoyenneté ne se réduit pas à ce premier niveau, mais à ce premier niveau vous le voyez, le savoir et le langage jouent un rôle central. Pas de possibilité d'avoir des droits si l'on n'a pas le moyen de passer du « droit de » au «droit à ».

2. Deuxièmement, la citoyenneté, et vous l'avez rappelé tout à l'heure, c'est avant tout une citoyenneté politique. Il ne peut pas y avoir de droits s'il n'y a pas en même temps, peu ou prou, un exercice du pouvoir. Est citoyen celui qui exerce le pouvoir. Et vous voyez que nous ne sommes pas encore vraiment citoyens et que la question est toujours devant nous, et que cette vraie question se pose aujourd'hui sous des formes tout à fait nouvelles qui traversent nos classes quelles qu'elles soient. Organiser une élection de délégués en $\mathrm{CM} 2$ pour leur faire faire l'apprentissage de ce que c'est qu'un délégué, c'est un problème que je ne connais pas parce que je n'ai jamais enseigné au CM2, mais c'est un problème que l'on rencontre de la même manière lorsqu'on souhaite organiser l'élection des délégués dans une Assemblée Générale d'étudiants en grève.

Il y a quelque chose de commun dans tout ça et c'est ça finalement exercer le pouvoir politique, c'est avoir cette capacité. Et là aussi, comment transmettre?

Pour la citoyenneté dans sa dimension politique, dans sa dimension accession au pouvoir, il faut donner le savoir et le langage. Mettez n'importe quel jeune devant un discours politique et essayez de décoder avec lui ce discours politique... pour lui, c'est un discours étranger. Donc, le problème est de savoir comment on va transmettre un savoir qui permette à ces jeunes d'avoir un discours politique ; car la politique, c'est noble. C'est une question que je pose parce qu'à mon avis si on n'en reste qu'au niveau 
des droits, on ne donne pas cette dimension-là et il va manquer quelque chose si on veut donner toute sa pleine dimension à la citoyenneté.

5 3. Condorcet avait commencé la réflexion en disant: la citoyenneté est totalement virtuelle si on ne donne pas les moyens de cette citoyenneté, à commencer par l'instruction. Il faut aller plus loin, la citoyenneté (et c'est la démarche qu'avait commencée Condorcet et qu'il s'agit de poursuivre) la citoyenneté n'est rien si sur le plan de la vie, économique, sociale, humaine, les individus ne sont pas dans des conditions qui leur permettent d'exercer cette citoyenneté.

Donc, cette dimension qui existe maintenant, c'est qu'il ne peut pas y avoir de citoyenneté si elle n'est pas aussi sociale. Ces trois dimensions que l'on décline en permanence, sans jamais bien les séparer ni les identifier sont fondamentales. Parlons par exemple du droit à la sécurité sociale. Le droit à la sécurité sociale c'est le «droit de ", c'est aussi un "droit à ». C'est une capacité à construire ce droit dans la solidarité avec les autres... Où est le langage, où est le savoir qui est transmis à ce sujet ?

Combien de jeunes je vois sortir à vingt ans passés ne sachant rien de la manière dont s'organise la solidarité dans notre société ? Pourtant, ils en sont partie prenante. Voilà donc, je pense ce que sont les 3 niveaux. On m'a demandé un peu de lancer le débat. On peut décliner à partir de là toute une série d'éléments où langage et savoir peuvent participer de la citoyenneté dans toutes ses dimensions.

arc COUTTY

Je vous remercie. Est-ce qu'en effet cette question du langage et de la possibilité, grâce au langage, d'accéder à la citoyenneté n'est pas, comme dit monsieur, sociale en son fond?

\section{Pierre BOUTAN}

Je crois que, d'un certain point de vue, tout le monde sait bien que tout est social, la question c'est de voir le rapport. Ce ne sont pas simplement les droits individuels, je crois que là-dessus l'histoire de l'évolution des déclarations des droits de l'Homme et du citoyen montrent à chaque fois que c'est une étape nouvelle... Il y a forcément aussi des droits collectifs. Mais du point de vue de l'école, moi je partirai comme le fait notre manifeste, je le dis parce que c'est une association récente et donc elle n'est pas connue de tous, elle est née en 1996-97 au moment où apparaît très clairement - c'est le moment du rapport Fauroux - au moment où apparaît très clairement que face au problème de l'école, il y a un certain nombre de forces sociales, idéologiques, politiques en France qui sont prêtes à chercher des solutions du côté de ce qu'on appelle le libéralisme ; c'est-à-dire, par exemple, des écoles différenciées selon les lieux, selon les origines sociales, l'appel aux capitaux privés pour financer les universités par exemple. En soi, ce n'est pas quelque chose d'extraordinaire, encore faut-il que le contrôle soit toujours celui de la fonction publique d'enseignement, par exemple. Toutes ces données ne sont pas si lointaines, et bien entendu, ce qui se passait en 1997 est toujours valable en 1998. Alors cette association, qui est née à partir d'un manifeste, regroupe le chercheur qui s'intéresse aux questions de l'éducation, mais aussi le praticien. La fierté de notre association, c'est justement de pouvoir regrouper et s'efforcer de faire travailler ensemble ces deux pôles importants de la réflexion au niveau de l'école - ce n'est évidemment pas exclusif - tous ces gens-là, se sont posé des questions simples :

- Comment se fait-il que, dans notre société, avec cette école que nous avons avec ses traditions, comment se fait-il alors qu'on voit bien qu'elle a fait des progrès au niveau du nombre d'enfants et de jeunes qui font des études longues, comment se fait-il qu'il existe 
aujourd'hui de telles difficultés pour un certain nombre d'enfants et comment se fait-il que la démocratisation recule au niveau de l'enseignement supérieur? Bien entendu, ceci a comme conséquence inévitable que se pose la question de savoir comment on va, comme le dit notre titre, notre label « défendre et transformer l'école pour tous ». La défendre parce qu'elle a des bases, la transformer parce qu'à l'évidence elle ne répond pas à ces besoins-là. Et tout cela évidemment postule un certain nombre de réflexions, de travaux de recherche, de relations fortes entre les praticiens et les chercheurs et au-delà tout l'ensemble du corps social et c'est ce qui c'était traduit par un colloque, je crois important, avec 500 personnes, 80 communications, qui s'était tenu en 1997 à Marseille. Au terme de cette étape, nous avons engagé de nouveaux travaux parmi lesquels un colloque qui aura lieu l'année prochaine sur le thème : «Quelle culture commune? ». Car, c'est un des grands problèmes que nous avons à résoudre dans la situation scolaire et je crois que c'est un problème de citoyenneté. Qu'estce qui fait la communauté ? Alors, sur ces bases-là, moi je crois que les problèmes qui sont posés sont des problèmes qui concernent chacun d'entre nous car dans les actes d'enseignement, il y a le choix entre accroitre les différences et les laisser se développer, ou au contraire chercher à les combattre. Dans ce cadre là, et c'est plutôt le linguiste qui interviendra, cette fois-ci un peu plus personnellement, la question de la langue je crois qu'elle a été assez abondamment traitée au cours de ce colloque, se pose avec toutes ses difficultés.

- Langue commune ou langue unique ? C'est une grande question. Pour le moment, ce qui fait problème, je crois, c'est que nous travaillons trop avec l'idée de langue unique. Et je crois que cela ressortait assez clairement de toute une série d'interventions, ce qu'il faut garder c'est la langue commune, mais montrer la diversité des possibilités dans ce domaine, et je crois que cela recouperait un certain nombre des soucis que nous avons évoqués. Alors évidemment, une fois que l'on a établi des principes, il faut après les traduire dans la réalité et mesurer les contradictions qui existent et qui ne sont pas simples à gérer. On évoquait tout à l'heure le cas des langues régionales, il y a aussi les langues de l'immigration, il y a aussi les langues étrangères. Dans ce domaine-là, comme on le sait, les emplois du temps ne sont pas extensibles et donc il y a de vrais problèmes de remise en cause, de questionnement sérieux ; je ne pense pas que l'on puisse avoir une position conservatrice de principe mais il ne faut pas oublier qu'il est illusoire de s'imaginer que l'on peut changer les choses sans tenir compte de ce qui s'est passé jusqu'à maintenant. Les traditions ne sont pas seulement négatives. Il faut en tenir compte pour les changer.

\section{Marc COUTTY}

Merci, langue commune, langue unique... Peut-être aussi faut-il s'interroger sur l'apprentissage de la langue, qu'elle soit commune ou unique... Comment peut-on apprendre une langue commune à l'école? Qu'est-ce que vous en pensez, Madame? Vous représentez je crois la FCPE, Madame Le Dain?

\section{Madame LE DAIN}

Oui bonsoir, je vous remercie de m'avoir invitée à cette réunion, je parle là du point de vue d'une fédération de parents d'élèves qui, comme le rappelait à l'instant Pierre Boutan a des traditions; qui a cinquante ans maintenant et qui a accompagné l'école depuis très longtemps et qui s'est toujours positionnée par rapport à l'école comme un groupe de parents d'élèves qui refusent l'idée que l'éducation puisse être un outil de consommation. On a des principes, c'est la laïcité, c'est la gratuité pour l'égalité des chances, la gratuité pour l'égalité de tout le monde et de tous les enfants face à l'éducation et pour que tous puissent se développer par rapport à leurs propres 
compétences. Et donc, c'est l'idée fondamentale, l'on refuse que l'accès à l'éducation soit un outil de consommation. En fonction de ses moyens - c'est un refus du libéralisme d'une certaine manière - chacun vient puiser ce qu'il peut y trouver. C'est l'idée que l'école doit pouvoir permettre à chaque enfant de développer son potentiel de compétences, son potentiel de talent au mieux de ce que l'histoire, histoire générale de notre pays, et histoire personnelle, lui permettra de le faire. Donc, c'est ça l'ambition de la FCPE et je crois que c'est également l'ambition de l'école; on s'est toujours retrouvé avec les mouvements d'enseignants. Et le thème d'aujourd'hui, savoir, langages et citoyenneté, interroge sur la langue commune à travers plusieurs champs.

Un premier champ, et je vais peut-être être un peu provocatrice, face à des enseignants, mais c'est peut-être notre rôle de parents d'élèves aussi, dans un colloque comme celuilà, est celui de l'intervention sur le contenu de ce qui est enseigné aux enfants, même si dans l'école nous nous gardons bien de rentrer dans ce débat. Mais c'est la façon dont les enfants très vite, très tôt, vers dix ans, onze ans au collège, sont amenés à regarder la langue non pas comme un outil pour se révéler eux-mêmes à eux-mêmes en se racontant, en racontant leurs idées, leurs projets, leurs rêves, leurs espoirs. A travers tout un code de formalismes on leur apprend à décoder la langue des autres, ce qui, en soi, est intéressant, en soi est utile, parce que c'est par ce biais là que l'on va approcher la culture commune à nous tous. Mais pour certains enfants, qui chez eux n'ont pas eu l'ensemble des codes préalables, l'école aujourd'hui en ce qui concerne l'enseignement des savoirs et des langages est trop formaliste. C'est un point sur lequel je souhaitais insister ce soir. Mais c'est le rôle de l'Education Nationale et des pédagogues de définir le contenu des programmes et je n'irai pas plus loin.

11 Mais un autre rôle de l'école, à notre avis n'est pas seulement l'enseignement des savoirs ou l'enseignement des langages, c'est aussi l'enseignement de la citoyenneté.

Et l'enseignement de la citoyenneté, c'est l'enseignement de comportements personnels et l'apprentissage de comportements sociaux et de comportements globaux. Là, par rapport à cet apprentissage-là de comportements globaux et de comportements sociaux, il y a plusieurs points : l'élection d'élèves délégués par exemple, en primaire, n'est pas de droit, elle n'apparaît qu'à partir du collège et là encore, même si les élèves sont élus délégués de droit, en pratique, la formation à la citoyenneté, c'est-à-dire la formation à la prise de parole, la formation à l'audace de la prise de parole dans un groupe d'adultes qui est le conseil de classe et face à un groupe d'enfants qui est la classe, cette formation est très loin d'être dispensée partout. Elle relève souvent d'un véritable travail de fond soit de quelques enseignants, soit d'un principal, soit de parents d'élèves qui sur de nombreuses années, travaillent, labourent le champ du Conseil d'Administration des établissements scolaires pour obtenir cette formation à la citoyenneté sur les élèves délégués, alors même que l'élection est obligatoire. Ce qui veut dire que la République aujourd'hui, en ce qui concerne l'éducation à la citoyenneté et la citoyenneté comme outil de développement des jeunes et d'une société future, l'a intégrée en tant que concept mais pas en tant que projet. Et le passage du concept au projet, je crois qu'on est en train de le vivre et je suis d'autant plus contente qu'un colloque se soit organisé sur ce thème, savoir, langages et citoyenneté pour aboutir à ce que l'on envisage de manière extrêmement claire et extrêmement forte dans les établissements scolaires, y compris en primaire, l'idée que des élèves puissent au fond de leur vie scolaire, et pas toujours les mêmes, apprendre à parler, à parler aux autres, à entendre les autres, à faire parler les autres, qu'ils possèdent les outils d'une culture commune et de médiation. Les conflits sont des choses qui peuvent devenir 
dramatiques à partir du moment où ils ne sont pas énoncés. Or l'énonciation des conflits, leur transformation en paroles c'est quelque chose qui n'est pas facile, qui n'est facile pour personne, même pas pour les adultes, nous le savons tous, mais qui est encore plus difficile pour les enfants dont on ne connait pas, les uns et les autres, l'histoire personnelle, dont on ne sait pas si chez eux, ils sont écoutés, entendus, appréciés ou au contraire désavoués. Et je crois que l'école a pour fonction, de la maternelle jusqu'au lycée, de permettre à des enfants et à des adultes de devenir des médiateurs de conflits. Cette fonction là, de médiation, passe forcément par le langage et ce langage-là passe par l'apprentissage de savoirs qui sont des savoirs communs qui sont des méthodologies communes et c'est là qu'on retrouve également le rôle de ces sciences qui sont apparues depuis fort longtemps mais que l'on voit aujourd'hui percer dans l'éducation : la psychologie, la sociologie, la psychologie du comportement... On voit à quel point la mise en place est lente de ces outils de médiation à l'intérieur de l'école.

Merci, Monsieur Antonini, de la Ligue des Droits de l'Homme...

J'ai pris l'habitude quand j'interviens dans un certain nombre de réunions de systématiquement dire : Ligue des Droits de l'Homme et du Citoyen. Je dirais presque parce que le combat des Droits de l'Homme existe constamment, c'est une lutte perpétuelle, mais, en France et nous sommes en France, je dirais volontiers que c'est le citoyen qui est en manque, plus que l'homme... Il y a toujours un balancier. On ne parle pas d'une chose, après on en parle trop. Maintenant vous ne pouvez pas ouvrir un journal, écouter la radio, voir la télé, sans qu'on ne parle de citoyenneté. Tout le temps, il y a tout de citoyen. Il y a la boulangerie citoyenne, tout ce que vous voulez qui est citoyen. C'est un peu agaçant, nous ne sommes pas en 1789 pour nous appeler citoyen entre nous, et mieux vaudrait moins en parler et l'appliquer dans les faits. L'appliquer dans les faits ça veut dire, en clair, que l'on doit savoir vivre ensociété ou plus exactement que l'on doit savoir vivre dans la cité. Ce qui suppose vraisemblablement un apprentissage moral, mais beaucoup d'autres choses. Et je partage assez le point de vue de ma voisine. D'abord parce que j'étais moi-même enseignant et je me rendais compte que, finalement, l'enseignement que nous donnions, je pense à moi-même, l'enseignement du français, par exemple, se fait avec des textes dont on peut dire que la hauteur morale est incontestable et à la limite, quand j'expliquais le Traité sur la tolérancede Voltaire, il y a là quelque chose qui est parfaitement d'actualité. C'est l'homme, ce n'est pas le citoyen et le citoyen, curieusement, c'est plutôt avec d'autres écrivains du XVIII ${ }^{e}$ qu'on pourrait en parler; mais j'ai toujours fait l'expérience que dans un lycée, si personnellement j'avais insisté sur Montesquieu et d'autres, beaucoup de mes collègues escamotaient le problème, comme si, être professeur de Lettres, était quelque chose qui ne prédisposait pas forcément à parler du citoyen. Autrement dit, notre mission était de former un homme mais pas forcément un citoyen. Je crois que c'est là quelque chose qui est très grave surtout quand on s'adresse à des adolescents.

On parle beaucoup de civisme. Dès qu'on parle par exemple de politique, il y a cette sorte de retrait de la part de tout le monde, y compris des adolescents qui fait que le mot " politique » est un mot banni, horrible, honteux. Or je dois signaler quand même que si le mot civisme est fondé sur une expression latine, politique après tout, c'est fondé sur la ville... Il est curieux de voir cette dérive qui fait que le civisme est fort bien 
porté mais que la politique est mal vue et que dès qu'il y a des manifestations de lycéens pour réclamer... davantage de savoir, souvent, et bien, si l'on parle de politique, ils s'enfuient précipitamment. Je dirais volontiers qu'au fond, et c'est un petit peu ce que nous avons fait nous-mêmes, la ligue des droits de l'homme et du citoyen n'a jamais craint de dire : nous sommes des politiques... pas au sens politicien du terme. Et la réhabilitation de la politique n'est pas simplement une question de vocabulaire.

\section{Marc COUTTY}

Je crois que ça va faire une parfaite transition, Madame Lazerges...

\section{Christine LAZERGES}

Oui, merci Pierre, parce que tu termines très exactement par ce par quoi je voulais commencer. Je me sens un peu ennuyée de parler ici à des enseignants de qualité qui traitent de savoir, langages et citoyenneté. Moi je ne suis que juriste, enseignante aussi, professeur de Droit et à l'heure actuelle député. Mais mon discours, forcément, ne va pas prendre la suite, rigoureusement, des discours des linguistes ou de ceux qui ont enseigné les lettres dans les lycées. Si l'on m'a demandé de participer et de dire quelques mots lors de ce débat c'est parce que Lionel Jospin m'a demandé, ainsi qu'à J.P. Belderic député-maire de Tourcoing, de lui remettre un rapport sur la délinquance des mineurs. Si je viens avec vraiment beaucoup de plaisir parler aux enseignants non pas de nos réponses à la délinquance des mineurs mais de nos observations, c'est parce que, très, très vite, nous avons pris conscience et nous l'avons écrit, que les propositions que nous allions faire seraient en très grande majorité des propositions qui concerneraient "l'amont» de l'acte délinquant posé et pas des propositions concernant «l'aval » de l'acte délinquant posé. Parce que, très rapidement, nous sommes partis d'une hypothèse qui est je crois largement confirmée, étayée, qui est celle d'un déficit de la socialisation aujourd'hui. Et bien sûr nous avons été amenés à consacrer un long chapitre à l'école. Comment pouvait-il en être autrement puisque, parlant de socialisation, il était évidemment impossible d'éluder, ou de passer vite, sur ce lieu de la socialisation privilégié qu'est l'école?

Ce sont des heures et des heures qu'un enfant, de la toute petite enfance jusqu'à l'adolescence, passe dans le milieu scolaire. Alors que dire de ce qui est fait pour la socialisation pour l'apprentissage de la loi dans l'école? Du langage qui est utilisé pour cela? De la place qui est faite et de ce qui est dit de la citoyenneté ? Je vais rejoindre beaucoup de ce qui a été affirmé tout à l'heure; il y a un discours sur les droits qui devient classique, mais qui très souvent, malheureusement, et même la Convention Internationale des Droits de l'enfant nous incite à commettre cette erreur, n'est pas doublé d'un discours sur les devoirs. Or si j'avais à privilégier la socialisation et l'apprentissage de la loi, je privilégierais le respect d'autrui et le respect d'autrui dans l'apprentissage des devoirs et des droits et j'insisterais très fortement sur l'accès aux droits. Cela a été évoqué implicitement tout à l'heure. Car comment inculquer ou apprendre, même par les méthodes pédagogiques les plus actives, ce que peut être le devoir d'un citoyen dans une société où tant d'enfants ont le sentiment que de toute façon quand ils seront adultes, ils n'auront accès à aucun des droits dont on leur parle? Quand ils sont sans espoir sur l'accès aux droits? C'est pour ça que je pense qu'il faut intimement lier le discours sur les droits au discours sur les devoirs et le discours sur le contenu des droits au discours sur l'accès aux droits. C'est un très lourd travail et l'une des 135 propositions de notre rapport consiste à dire fortement qu'il faut que, dès la première année d'école pré-élémentaire, le programme comporte un travail très 
sérieux sur l'apprentissage des droits et des devoirs. Et que ce travail se fasse en liaison avec les familles. C'est une autre proposition forte de notre rapport qui est reprise par une circulaire du début du mois, une circulaire interministérielle qui a fait que le budget de Martine Aubry et le budget de Claude Allègre et le budget de Claude Bartolone et le budget de Elisabeth Guigou à la justice, comportent une ligne budgétaire sur la mise en place de lieux d'apprentissage, pour les parents, de la parentalité. Des lieux d'écoute et d'apprentissage pour les parents qui ne doivent pas forcément être dans l'école, mais qui doivent fonctionner en liaison avec elle. Je pense que dans une société où tant de parents sont en difficulté, sur la simple façon d'être parent, il est indispensable que les enseignants, et je sais que c'est une nouvelle charge, ne s'adressent pas seulement aux enfants, mais trouvent les façons, la façon, de s'adresser aussi aux parents. Et puis, j'ajouterais qu'il m'est toujours apparu et alors maintenant ça me paraît presque scandaleux, que la place qui est faite au droit dans l'enseignement français ne peut plus rester ce désert. Je me suis amusée un jour, pour un texte que j'écrivais sur l'apprentissage de la règle de droit, pour des juristes, à comparer un certain nombre de manuels d'éducation civique. Je les ai trouvés pleins de choses magnifiques sur les déclarations des droits ou sur des droits comme le droit de propriété et rien de très concret. Vraiment des manuels remarquables. J'ai comparé des manuels pour des classes de quatrième. Remarquables pour des étudiants en troisième année de droit, mais qui m'ont paru assez largement inadaptés à ce que sont, sur ces questions-là, des enfants, des pré-adolescents ou des adolescents de classe de Quatrième. Rien de très concret, rien sur le vol, rien sur l'abus de confiance, rien sur l'escroquerie, quasi rien sur le mariage, sur le divorce qui sont des choses qui parlent à des adolescents.

Mais en revanche : "Tous les hommes naissent et demeurent libres et égaux en droits » a été développé, décliné de toutes les façons. Et à mon sens, on ne peut décliner les grandes déclarations que si on a un petit bagage concret, un petit bagage juridique concret. Je ne suis pas tout à fait sûre que les enseignants l'aient d'ailleurs, puisqu'ils nous sollicitent très fortement pour intervenir dans leurs classes sur la manière dont fonctionne la justice, sur ce qu'est le droit civil, le droit pénal. Les programmes, je le sais, vont avoir tendance à être allégés et non pas à être alourdis. La France est l'un des seuls pays au monde où l'on enseigne la philosophie, et je m'en félicite, mais c'est un des seuls pays au monde où l'on fait aussi peu de droit, pour ne pas dire pas de droit, avant d'arriver aux études supérieures... et ça c'est une catastrophe.

\section{Jean-Pierre OBIN}

Je vais continuer. Moi je suis pour le droit à l'école mais aussi pour la morale. Bien. Je vous rappelle le mot d'O. Reboul «la morale commence hors de la vue du gendarme tandis que le droit évidemment est d'abord fondé sur la crainte, dit-il, la peur du gendarme ».

Mais à propos du gendarme, je voulais dire que ça se trouve bien que je parle juste après Christine Lazerges parce que moi je suis chargé cette année par Elisabeth Guigou et Ségolène Royal d'un rapport sur l'enseignement dans les prisons. Et ce que je découvre dans les prisons en allant enquêter avec mes collègues de l'Inspection Générale des services judiciaires me surprend, m'étonne parce que je ne connaissais pas du tout. Je n'avais jamais fait cette expérience et je n'avais jamais été enquêter dans le milieu carcéral. Et la question qui se pose notamment c'est, face à ces jeunes criminels de 16 ans - on n'emprisonne pas pour des problèmes de délinquance mineure - de savoir qui se préoccupe de l'éducation de ces jeunes en prison. Ils sont en face d'une 
multiplicité d'interlocuteurs, notamment les jeunes mineurs, pour lesquels il y a un certain nombre de règles, en prison, qui contraignent l'administration pénitentiaire à les entourer par un certain nombre de services... Ils ont en face, d'une part des enseignants, ce sont des professeurs de l'enseignement secondaire, une partie d'instituteurs spécialisés, qui reçoivent une mission d'enseignement. L'enseignement est un droit en prison et cet enseignement est dispensé de la même manière que pour tout public d'un âge équivalent. C'est-à-dire que l'on fait des cours de mathématiques, de français, etc.. En tout cas, les enseignants que nous avons interrogés ne se sentent pas en charge de l'éducation des jeunes en question, et, certainement pas, de l'éducation morale en particulier.

21 Deuxièmement, la Protection Judiciaire de la Jeunesse (anciennement Education Surveillée; en 1970 l'éducation surveillée change de nom devient Protection Judiciaire de la Jeunesse et l'on passe d'une conception éducative à une conception de mineur à protéger). La P.J.J., aujourd'hui, n'est pas dans les prisons. Elle est à l'extérieur des prisons. Et lorsqu'un éducateur de la P.J.J. vient voir un jeune dont il avait la charge avant qu'il soit emprisonné, il est soit au parloir, comme un visiteur normal, soit il peut le rencontrer dans une salle aménagée à cet effet. Mais il est un visiteur extérieur. L'administration pénitentiaire a créé un corps d'éducateurs, pour prendre en charge la dimension socio-éducative dans les prisons. Et ce corps d'éducateurs est en train de se transformer en service de probation et d'insertion pénitentiaire. J'ai rencontré un de ses « responsables ». Le mot d'éducateur semble banni aujourd'hui. Ils se présentent comme des travailleurs sociaux chargés d'être les intermédiaires entre les différents interlocuteurs internes et externes et les prisonniers; mais ils semblent refuser globalement, en tout cas institutionnellement, la mission éducative. Alors, il y a les religions qui traditionnellement assument ou doivent assumer une tâche d'éducation morale. Les aumôniers viennent toujours dans les prisons, mais la majorité des jeunes prisonniers sont de religion et d'origine musulmane. Et d'après ce que l'on nous a dit, un certain nombre d'imams refusent de venir et d'aller les voir, parce que ce sont des délinquants et des criminels, justement. Aussi, ils se retrouvent face à une espèce de vide d'interlocuteurs, qui est comblé çà ou là par un certain nombre de personnalités : des surveillants volontaires, quelques enseignants qui acceptent, ou volontairement posent, le dialogue sur le plan moral. Et en particulier sur le plan de ce qui a été fait et de ce pourquoi le jeune est là, en prison, de l'acte dont il est l'auteur et qui l'a amené à une peine parfois lourde. Mais, institutionnellement, l'acte par lequel le jeune criminel ou délinquant arrive en prison est quelque chose qui n'est pas pris en compte à l'intérieur de la prison. On est censé ignorer nous dit un enseignant pourquoi ils sont là. Alors même que ce qui est important, grave pour la vie de ces jeunes, c'est évidemment l'acte qu'ils ont commis et dont ils vont avoir, quoi qu'ils fassent, à assumer la responsabilité pendant toute leur vie. Alors, voilà peut-être un des domaines qui me semble bien à la marge de notre société ; qui n'est pas forcément représentatif de tout ce qui se passe ; mais ce qui m'intéresse c'est comment, aujourd'hui, la mission éducative peut être assumée par l'ensemble des professeurs dans les écoles. Comment assurer donc la prise en charge des problèmes éducatifs des jeunes d'aujourd'hui ? Il faut admettre que, malheureusement, l'éducation nationale ne nous donne pas la formation qui serait nécessaire à cette prise en charge.

\section{Marc COUTTY}

Je vous remercie. Je vais passer la parole à la salle mais avant, si vous me permettez, de dire quelque chose... C'est une question, c'est une réflexion plutôt que je voudrais vous 
faire à vous messieurs, notamment. Dans Le Monde, il y a je crois un mois ou deux, a été publiée une très, très intéressante étude d'un institut d'opinion, la SOFRES je crois, ou l'IFOP, commentée par des journalistes du Monde sur les rapports des enseignants avec les différentes transgressions des lois. On s'apercevait de cette façon, incidemment, qu'il n'y a pas que les élèves qui soient en situation de transgression mais qu'on avait, par exemple, au hasard, sous réserve de vérification du chiffre, on avait $40 \%$ des enseignants qui avaient déjà, dans leur vie, au moins une fois usé de produits stupéfiants. On s'apercevait que les enseignants ne considéraient pas particulièrement comme un défaut ou comme une chose répréhensible, de mentir quand ils y trouvaient avantage. Donc, je dis ça d'une façon un peu provocante, sachant que je suis devant un parterre vraisemblablement composé presque totalement d'enseignants, pour m'étonner un peu de cette, comment dire, rigidité... de cette verticalité, du propos moral de votre part sachant que ceux auxquels vous voulez confier cet enseignement moral, dont pour ma part je ne vois pas toujours très bien qui ils pourraient être, ont chacun, leur petite morale. Il y a peut-être, évidemment, une grande morale civique. Les dix commandements, revisités manière laïque. Mais je vois mal comment on pourrait demander à des gens qui eux-mêmes, ma foi, sont comme nous tous, avec leurs petits défauts, avec leur conception de la société, de ce qui est juste et de ce qui l'est moins, leur demander de s'ériger en moralistes.

\section{Jean-Pierre OBIN}

Je me suis fait très mal comprendre visiblement, parce que lorsqu'on parle de morale, on entend tout de suite: voilà quelqu'un qui est pour l'ordre moral, qui est un moralisateur.

Je sais faire la distinction entre les comportements qui sont mes comportements privés et puis ce que je pense être bon pour ceux qui sont en face de moi et ma responsabilité d'adulte par rapport aux enfants. Je crois que c'est cette part qu'il faut être capable de faire. A tout moment lorsque je suis dans une position d'éducateur, en plus dans une position d'éducateur institutionnel, je dois choisir entre les comportements qui sont les miens en privé, dans la famille, dans le cercle de mes amis etc... et ce que je pense bon pour ceux dont j'ai la responsabilité, et je dois toujours adopter envers eux, un comportement responsable. J'assume mes actes privés par rapport à ce qu'ils sont à la fois sur le plan moral et sur le plan juridique; pour autant je ne vais pas en faire un modèle et dire : «c'est comme ça que vous devez vous comporter parce que moi je me comporte comme ça en privé ». Il y a cette distinction à faire.

\section{Marc COUTTY}

Je vous remercie, je vais passer la parole à la salle.

Gilles BUI-XUÂN - (intervenant dans la salleJe voudrais poser une question à René Revol au sujet des projets ministériels concernant l'avenir des sciences économiques et sociales au lycée qui semblent poser quelques problèmes à notre ministre et qu'il voudrait remplacer, éventuellement par des exercices de droit.

\section{René REVOL}

$\mathrm{Tu}$ poses un sujet tout à fait sensible... Comme mon interlocuteur à ce sujet, Claude Allègre n'est pas ici, pour pouvoir éventuellement me répondre, je m'exprimerai d'une manière relativement limitée. Mais cela me permet de rebondir sur une question. Peuton enseigner la citoyenneté ? Peut-on enseigner le droit? Peut-on enseigner la morale? Moi je pense que oui, mais ces trois choses ne sont pas sur le même plan. Par exemple, aujourd'hui, il y a des éléments de droit qui sont présents dans les 
programmes d'histoire dans les programmes de sciences économiques et sociales, dans les programmes de philosophie. Et ces éléments ne sont pas abordés en tant que droit, c'est ça le problème que vous posiez Madame Lazerges tout à fait justement. La question c'est que lorsqu'un professeur de philosophie - je fais exprès de répondre par une autre discipline pour ne pas me sentir engagé directement - aborde la question du droit, il l'aborde à travers la façon dont la philosophie a pensé le droit ; c'est-à-dire une façon qui n'est pas normative. Une façon qui est critique. Non pas : le droit c'est la règle à laquelle il faut obéir ; mais : le droit c'est une construction de l'humanité ; c'est une création de l'humanité. Donc, à partir de ce moment-là, nous avons la même démarche dans d'autres disciplines, en histoire, en sciences économiques et sociales. Donc, effectivement, le débat est ouvert. S'il s'agit aujourd'hui de transformer les professeurs de philosophie qui sont formés pour aider leurs élèves à atteindre un esprit critique, s'il s'agit de transformer les professeurs de sciences économiques et sociales qui sont formés pour aider leurs élèves à atteindre un esprit critique et idem pour les professeurs d'histoire, s'il s'agit de les transformer en "dispensateurs", pour les premiers, d'une morale, pour les seconds, d'un ordre normatif, et pour les troisièmes, de l'histoire des vainqueurs et non de celle des vaincus, naturellement, l'immense majorité de ces enseignants refuserait. Lorsqu'ils ont compris des projets comme ça - je ne dis pas, par là même, que ce sont les projets du ministre - mais lorsqu'un projet est compris comme ça par les enseignants, ces enseignants sont amenés à le refuser. En fait, ça permet de rebondir sur le débat que nous avons... Peut-on enseigner la citoyenneté ? La citoyenneté s'enseigne déjà; je provoque un peu exprès; elle s'enseigne déjà car elle s'enseigne d'une manière transversale dans toutes les disciplines. Parce que dans toutes les disciplines, la question de la place que nous occupons dans la cité est posée. On ne peut pas enseigner le français comme l'a expliqué Pierre tout à l'heure sans choisir entre Montesquieu et Condorcet pour le texte qu'on fait demain; pour savoir quelle dimension on donne. Par là même, on rentre dans l'apprentissage de la citoyenneté. Et là dessus les débats sont loin d'être clos. Cela signifie qu'il ne s'agit pas de transformer les disciplines qui existent aujourd'hui. Il faut au contraire les maintenir, les développer. Cela ne signifie pas développer leur horaire, mais les développer quant au contenu. Mais est-ce qu'il faut faire un enseignement spécifique de la citoyenneté, un enseignement spécifique de la morale? Je dois avouer que dans les débats actuels de la majorité des éducateurs que j'ai rencontrés, les enseignants doutent de l'efficacité de cet enseignement. On va vous enseigner ce qu'il faut savoir. Je sais, tout le monde le sait, que c'est la meilleure manière de dresser tout le monde contre cette morale, contre ce droit. Donc il va falloir travailler, travailler au-delà du: «il faut». C'est-à-dire essayer de voir par quel cheminement on peut permettre cet apprentissage de la citoyenneté, ensemble, dans un lycée, dans un collège, dans une école, etc... Voilà c'est une manière d'élargir, par rapport à ta question, sur un des débats qui a traversé toutes nos interventions d'une certaine manière.

\section{Une personne dans la salle}

En ma qualité d'enseignante en ZEP en classe de perfectionnement avec un public d'élèves en difficulté scolaire qui ont entre 10 et 12 ans, révolus, je me suis beaucoup posé de questions à propos de la loi. Et justement, j'ai eu beaucoup d'intérêt à entendre les deux personnes qui ont fait référence à la loi dans la société. Je crois que l'on nous parle beaucoup de citoyenneté mais ce qui nous manque aussi c'est un contenu au niveau légal. C'est-à-dire que l'on parle des conseils de classe, de délégués des élèves ; 
on parle de prise de parole; on parle du bien, du mal. Mais quand un élève fait effectivement une bêtise, une grosse... style attouchement sexuel d'un enfant vis-à-vis d'un autre, ou bien vol, ou bien... Je crois que la référence de l'école doit être la référence de la loi dans la société. Qu'est-ce qui arrive dans un cas comme ça dans la société? Et là je trouve qu'un enseignant est tout à fait démuni. C'est-à-dire qu'effectivement, de temps en temps, en ZEP il y a les policiers qui viennent nous expliquer. Mais je trouve que c'est important qu'avant le collège, avant le lycée, avant la Fac, les élèves aient une prise de conscience que tel comportement peut induire telle sanction. Cela fait partie effectivement de la citoyenneté. Parce que la citoyenneté c'est la prise de parole, les droits, mais aussi les devoirs envers les autres. C'est un garde-fou, je pense, pour eux, de savoir qu'en effet, il y a une loi, il y a une sanction aussi.

\section{Marc COUTTY}

Vous voulez dire quelque chose?

\section{Christine LAZERGES}

Oui, je voudrais dire juste un mot, répondre à Madame. Mon équipe de recherche universitaire a fait un travail sur l'apprentissage de la loi et le travail de terrain s'est fait dans quelques collèges et lycées de Montpellier et de sa région. Et nous avons été nous-mêmes sidérés de l'incapacité, pour beaucoup d'adolescents, de simplement savoir si ce qu'ils font fait partie du permis ou de l'interdit. C'est-à-dire que, pour eux, il y a des vols qu'ils ne qualifient absolument pas de vols. Il y a des usages de produits dont ils n'imaginent pas un instant que ce n'est pas autorisé. Je veux dire par là que si vous approchez le droit, par Montesquieu que j'aime beaucoup, je crains que, en ZEP, ce soit quand même une approche compliquée. Il y a quelques années encore, les familles complétaient cette approche par de grands auteurs. Un travail constructif sur la loi se faisait au sein de la famille. Mais sachez qu'il ne se fait plus. Du moins qu'il ne se fait plus dans beaucoup de cas. Et c'est cela le drame. Si des apprentissages qui se faisaient au sein de la famille ne se font plus au sein de la famille et s'ils ne se font plus nulle part, on va à la catastrophe. Et c'est formidable de savoir que Montesquieu est le père de la séparation des pouvoirs pour un adolescent. Mais c'est quand même mieux de savoir ce qu'est le vol et ce qui se passe quand une réponse est apportée par l'école ; car une réponse peut être apportée par l'école, sans poursuite pénale forcément. Mais qu'est-ce qui se passe s'il y a une poursuite pénale? Et si le professeur ne sait pas la différence entre un magistrat du siège et du parquet, et s'il ne sait pas expliquer ce que la société a mis en place à cet égard, même maladroitement, c'est quand même très ennuyeux.

\section{Marc COUTTY}

Merci. Excusez-moi, une toute petite remarque en fonction de ce que vous venez de dire, madame. En tant que journaliste spécialiste d'éducation, je connais beaucoup d'enseignants. On les suit ; on va dans les écoles; on va dans les collèges; on va dans les lycées. Moi, ce que je sens actuellement c'est que les enseignants se plaignent un petit peu que l'on charge trop la barque. Qu'on leur en demande beaucoup. On leur demande d'instruire, on leur demande de transmettre un savoir, ce qui est légitime, ce qui est leur fonction première... On leur demande aussi maintenant, un peu comme vous venez de le dire, de se substituer aux familles, de prendre en charge toute cette détresse, toute cette misère d'une partie de la population jeune du pays, parce que toutes les autres institutions n'y arrivent plus. Mais est-ce que vous ne croyez pas qu'en 
chargeant ainsi la barque, on ne risque pas, à un moment ou l'autre, de les saturer et les décourager?

\section{Pierre ANTONINI}

Trois secondes simplement avant que vous ne preniez la parole à propos de ce que vient de dire Christine Lazerges sur le vol. C'est vrai qu'il faudrait peut-être le dire, mais toute approche est nécessairement critique. Avec mes élèves j'ai eu à expliquer comment en 1943 il n'y a pas si longtemps, les femmes avaient été exécutées en France, pour complicité d'avortement. Maintenant l'avortement est légalisé ; donc tout délit, à la limite, est quelque chose qui est évolution.

Jacques GLEYSE - (intervenant dans la salle)Moi j'ai l'impression quand même qu'il y a des niveaux de compréhension de la citoyenneté. J'entends quand même beaucoup, et je suis désolé, vous l'avez souligné plusieurs fois, mais malgré tout j'entends beaucoup quand on parle de citoyenneté se développer un discours de type néo-colonialiste puritain. Moi j'entends fréquemment tenir un discours puritain. Vraiment. Je n'entends pas tenir un discours critique... Se demander pourquoi la philosophie reste dans les classes terminales et ne descend jamais plus bas. Pourquoi les thèses de P. Bourdieu sont enseignées très tardivement pendant la scolarité, et pas beaucoup plus tôt, et ne seront plus enseignées avec la suppression des sciences économiques et sociales? Pourquoi ne sont jamais mises en place les positions de pédagogie institutionnelle? Je pense à des choses comme ça... Effectivement, le problème du vol, bon c'est un problème. Mais jusqu'où est-on volé ? Moi je rediscute ce problème. Jusqu'où on vole ? Pendant la seconde guerre mondiale il y a sûrement eu beaucoup de vols qui ont été faits de très bonne manière et peut être même des meurtres qui ont été de très bonnes choses, durant la seconde guerre mondiale, dans ces conditions-là, particulières. Par contre le problème de la critique c'est ça qui est essentiel, le problème des positionnements et de la position critique. En tous les cas pour moi, la citoyenneté ça ne sera jamais un puritanisme pur et simple... J'entends quand même beaucoup cette position-là dans tout ce qui est dit.

\section{Marc COUTTY}

D'autres interventions, Monsieur tout en haut de la salle je vois que quelqu'un a levé la main.

Salim MOKKADEM - (intervenant dans la salle)Je voulais dire simplement que dans les prisons on vient actuellement, ça fait dix ans que c'était demandé, d'instituer le parloir sexuel, que le respect de la personne privée enfin, n'existe pas... On fait tout pour dépersonnaliser par les numéros d'écrou. Que le taux de mortalité y est trois fois plus important qu'ailleurs. C'est pas simplement au vu de l'état de santé des personnes entrant, mais des conditions de vie. Faire de la morale à des gens qui sont en prison ce me semble quelquefois un peu indécent. Je serais d'accord avec mon collègue Gleyse pour dire: puritain et néo-colonial. Je vous renvoie à la lecture de Surveiller et Punirque vous avez certainement lu mais qui n'a pas eu l'impact qu'il aurait dû avoir au niveau des politiques carcérales. Le rapport à la citoyenneté est pour vous, à vous entendre, de l'ordre de la sanction, de la loi... J'ai l'impression, tout de même, je m'adresse à la juriste et non plus au député, que vous confondez un petit peu légitimité et légalité et que d'une certaine manière, s'il est nécessaire de faire du droit comme de la philosophie, il faut savoir qu'on commence à faire de la philosophie juridique, ou de la philosophie du droit quand on remarque que le droit c'est d'abord la gestion d'un rapport de force et que, d'une certaine manière, c'est un domaine qui exclut beaucoup 
plus qu'il n'inclut... Bien sûr, il confère des avantages mais lorsqu'on est justement en position symbolique, je renvoie à Bourdieu, en position de pouvoir s'appuyer sur ces avantages symboliques. Mais aujourd'hui ? Je ne vais pas rappeler la question des sanspapiers, mais quand vous dites, je le dis sans agressivité aucune, avec un peu de naïveté appuyée, que les jeunes ne savent pas ce que c'est qu'un vol... je me demande quels jeunes vous fréquentez, si vous êtes déjà allée dans un collège, comment vous travaillez. Et j'ai envie de dire comme ça, parce que l'on pourrait en discuter par après, de manière plus scientifique, en prenant des textes... que lorsque les - on va mettre des formes - lorsque les SS, les officiers SS visitaient les camps de concentration tout était propre, il y avait de la musique, tout le monde était bien nourri. Peut-être quand vous allez avec votre casquette de chargée de mission ou de député, tout va bien. Mais le problème, c'est qu'il faudrait être un peu plus anonyme et regarder du côté de ceux qui sont exclus le rapport qu'ils ont à la citoyenneté. On ne peut pas être citoyen dans une société qui utilise de la violence symbolique. Qui exclut, qui est particulièrement agressive, voire arrogante, puritaine, qui dit des banalités du style : « il faut rétablir la morale » sans savoir laquelle; qui ne réfléchit pas à la contradiction en disant : « il faut faire de la philosophie et du droit en même temps » alors que la philosophie et le droit n'ont pas la même histoire ; n'ont pas les mêmes rapports institutionnels ; n'ont pas les mêmes rapports économiques, les mêmes rapports de force de représentation etc.. Autrement dit, il me semble que vous parlez un petit peu de la citoyenneté en nantie du pouvoir, symbolique ou autre, politique, et qu'il vous manque peut-être, cette distance critique, cette dialectique qui vous permettrait de penser la citoyenneté en termes de rapports de force simplement.

Christine LAZERGESMonsieur vous contestez quantité de choses... Que je sois nantie et que vous le soyez aussi c'est une évidence. Nous ne serions pas sinon cet après-midi dans cet amphithéâtre ensemble. Point de départ. Le mieux est d'en être parfaitement conscients.Deuxième chose, je n'ai pas utilisé, pas une seule fois le mot de «morale ». On pourrait en parler, c'est un autre débat, pas une seule fois.Troisième chose, nous essayons ici de travailler à la conjugaison entre le savoir, le langage et la citoyenneté et j'ai voulu prendre des exemples extrêmement concrets en vous parlant de ce qui pouvait être proposé pour les écoles pré-élémentaires mais je ne voudrais pas que vous pensiez que mon discours soit réducteur au point que seul le vol m'intéresse et seule la sanction du vol. Bon, sur ces sujets-là, on pourrait parler longtemps, ce n'était qu'un exemple. Je ne vois pas très bien, hormis les lectures que vous avez avancées et que j'ai faites aussi, je ne vois pas très bien où vous voulez en venir. L'apprentissage de la citoyenneté, bien entendu, a pour objet de faire de l'enfant un acteur de la société et pas un exclu de la société. Or qu'est-ce que nous constatons aujourd'hui? C'est que la société exclut plus qu'elle n'excluait, et, donc, nous essayons de réfléchir ensemble au moyen, de faire des êtres humains, le plus tôt possible, des acteurs. Et la citoyenneté, l'apprentissage de la citoyenneté est à mettre en relation avec l'apprentissage de la responsabilité. Moi, mon seul objectif quand je traite de ces questions, quand j'y réfléchis avec d'autres, c'est d'avancer un tout petit peu, de poser une toute petite pierre dans un sens qui permettrait à un plus grand nombre d'être acteurs de la société.

\section{Marc COUTTY}

Nous allons devoir arrêter le débat dans quelques minutes, je laisse la parole à Monsieur Revol qui me la demande depuis un moment et après je pense que, malheureusement, nous devrons conclure. 
Je pense que la brièveté de notre débat fait qu'il y a plusieurs niveaux de débat qui ont démarré, et qui ne peuvent pas se construire, et qui sont assez passionnants. Et même si parfois, les propos des uns ou des autres ont pu être compris d'une manière ou d'une autre, un peu vivement, je pense que ces débats doivent être poursuivis. Je suis d'une génération qui a connu des débats vifs, et je trouve que ça manquait un peu. Donc moi je suis pour qu'il se développe, ce débat.

Maintenant, je voudrais intervenir sur un niveau du débat, non pas sur tous les niveaux qui ont été abordés. Famille - Ecole. On ne va pas se repasser le «mistigri » pendant longtemps. Il y a une crise sociale, elle s'exprime dans la famille, elle s'exprime dans l'école et comme la famille ne l'assume pas, elle demande à l'école, et comme l'école ne l'assume pas, elle dit «c'est la famille ». C'est d'une certaine manière l'art de la famille et l'art de l'école qui cachent la crise de la société. Donc, on ne va pas s'en sortir dans cette discussion. Simplement, on a des acquis. Notre acquis notamment: moi je suis enseignant de l'enseignement public et laïque. Et pour moi, la laïcité c'est la séparation du public et du privé parce que c'est une façon de protéger la personne et c'est aussi une façon de permettre à chacun d'exercer sa citoyenneté. Donc, il faut faire attention. Je suis d'accord pour développer les réseaux, il y a beaucoup de réseaux à développer... Il faut faire attention quand, par exemple, les enseignants ont des informations personnelles sur les enfants qui les conduisent à avoir une attitude vis-à-vis d'eux qui permette de faire face à leurs problèmes tous les jours (il y a des centaines de cas), ils font attention, ils ont une déontologie, ils ne les mettent pas sur la place publique, ils les utilisent pour tenter de faire que l'acte éducatif, parce que c'est de ça qu'ils sont responsables dans l'école, ait une certaine qualité, au vu de la situation personnelle de l'enfant qui est en face. L'enseignant n'ira pas régler le problème personnel; il le transmettra aux institutions sociales lorsque le problème est grave ou à la famille. Que donc, cette séparation entre le public et le privé, et non pas la dissolution dans une sorte d'ensemble «sociétal ", où tout le monde réglerait le problème avec tout le monde, est une protection contre l'inégalité de l'ensemble sociétal en question. Parce qu'effectivement, aujourd'hui, si nous nous tenons à ce que les choses se fassent dans l'école c'est pour qu'il y ait justement l'égalité d'accès et que ce ne soit pas en fonction des familles et $\mathrm{du}$ réseau social dans lequel sont les familles que se règlent certains problèmes. C'est le problème de la médecine scolaire, c'est le problème de la psychologie, etc... Et là, je voudrais aller plus loin, pour aborder la question de la morale et du droit. Dans mon enseignement, j'ai toujours enseigné l'Etat. J'enseigne l'Etat. Pas comme un philosophe. Qu'est-ce que l'Etat qui est là ? Je suis amené à faire des territoires, des cartes et je n'oublie pas de faire des cartes judiciaires qui font partie de l'Etat. Naturellement, les élèves s'aperçoivent que la carte judiciaire ne correspond pas à la carte administrative. C'est pour vous montrer qu'on enseigne le droit quelque part. Et à chaque fois les élèves, devant ces fameuses "Cours d'Appel »: «Pourquoi elles sont situées dans ces bleds paumés? Pourquoi elles ne sont pas dans la capitale?» Elles sont d'Ancien régime. Et alors moi, comment je peux leur expliquer la justice, sans leur dire que la justice a gardé un caractère féodal ? Et comment je pourrai, à ce moment là, leur dire que le droit émis par cette justice est indiscutable ? Je ne peux pas le dire, et au contraire je ne cherche pas à le dire. Mais je veux dire qu'on n'est plus dans Montesquieu. On est dans un exemple concret. Il ne peut pas y avoir de connaissances objectives sans qu'il y ait un même temps une démarche critique. Cela 
c'est la tradition des enseignants. Cela c'est un vrai problème. Ce n'est pas facile à mener. Les deux côtés m'attirent, les deux choses en même temps. Voilà.

Merci, je vous remercie.

\section{ANNEXES}

Participants au débat

N.B.

1. Les noms des participants sont suivis de la qualité en laquelle ils sont intervenus ou de l'organisme au nom duquel ils parlaient.

2. Ne figurent dans cette liste que les intervenants invités et non pas ceux faisant partie de l'assistance (personnes présentes dans la salle).

Christine LAZERGES - Député

René REVOL - Cercle Condorcet

Marc COUTTY - Le Monde de l'Éducation

Pierre BOUTAN - Défendre et Transformer l'École pour Tous

Anne Yvonne LE DAIN - F.C.P.E.

Pierre ANTONINI - Ligue des Droits de l'Homme et du Citoyen

Jean-Pierre OBIN - Inspecteur Général de l'Éducation Nationale

\section{RÉSUMÉS}

Transcription du débat

transcription of debate

\section{AUTEUR}

\section{MARC COUTTY}

Journaliste au Monde de l'éducation 